\title{
Heating of the solar wind with electron and proton effects
}

\author{
Ben Breech*, Steven R. Cranmer ${ }^{\dagger}$, William H. Matthaeus**, Justin C. Kasper ${ }^{\dagger}$ and \\ Sean Oughton \\ *NASA Goddard Space Flight Center, 8800 Greenbelt Road, Greenbelt, MD 20771 \\ ${ }^{\dagger}$ Harvard-Smithsonian Center for Astrophysics, 60 Garden Street, Cambridge, MA 02138 \\ ${ }^{* *}$ Department of Physics and Astronomy, Bartol Research Institute, University of Delaware, Newark, DE 19716 \\ ¿Department of Mathematics, University of Waikato, Hamilton, New Zealand
}

\begin{abstract}
We examine the effects of including effects of both protons and electrons on the heating of the fast solar wind through two different approaches. In the first approach, we incorporate the electron temperature in an MHD turbulence transport model for the solar wind. In the second approach, we adopt more empirically based methods by analyzing the measured proton and electron temperatures to calculate the heat deposition rates. Overall, we conclude that incorporating separate proton and electron temperatures and heat conduction effects provides an improved and more complete model of the heating of the solar wind.
\end{abstract}

Keywords: turbulence, solar wind, electron heat conduction

PACS: 94.05.Lk, 96.50 Ci

\section{INTRODUCTION}

The supersonic solar wind accelerates away from the Sun through some combination of physical processes including gradients in gas pressure (from the hot, $10^{6} \mathrm{~K}$ corona) and wave pressure, as well as possible collisionless wave-particle interactions. The solar wind displays a highly non-adiabatic temperature profile, requiring some process(es) to provide additional heat sources. One possible, and successful, source of heating comes from magnetohydrodynamic (MHD) turbulence present in the solar wind [1]. An active MHD turbulent cascade [2, 3] transfers energy from the large-scale fluctuations down to small scales where kinetic processes dissipate the energy as heat.

With some exceptions, [e.g., 4], turbulence theories [such as 5, 6, 7, 8] often neglect electrons in favor of the protons as the protons help set the scales of interest. Neglecting electrons is justified in the case of the momentum content and the mass density of the solar wind plasma. However the internal energy content of the electrons is not negligible compared to that of the protons. Indeed, in the mainly collisionless solar wind, the various particle species (i.e., protons, electrons, and heavy ions) are not in thermal equilibrium with one another. The particles exhibit a range of different outflow speeds, temperatures, and velocity distribution anisotropies, particularly in regions with the least frequent Coulomb collisions $[9,10]$.

In this work, we seek to understand how electrons and protons conspire to heat the solar wind. For the case of proton and electron temperatures $\left(T_{p}\right.$ and $\left.T_{e}\right)$, we can write down evolution equations assuming a steady state, spherically expanding solar wind with a radially constant bulk flow speed, $U$, and a number density profile varying as $n \sim r^{-2}$;

$$
\begin{gathered}
\frac{\mathrm{d} T_{e}}{\mathrm{~d} r}=-\frac{4}{3} \frac{T_{e}}{r}+\frac{2}{3} \frac{1}{k_{B} n U} Q_{e}-\frac{T_{e}-T_{p}}{U \tau}-\frac{2}{3} \frac{1}{k_{B} n U} \nabla \cdot \mathbf{q}_{e}, \\
\frac{\mathrm{d} T_{p}}{\mathrm{~d} r}=-\frac{4}{3} \frac{T_{p}}{r}+\frac{2}{3} \frac{1}{k_{B} n U} Q_{p}+\frac{T_{e}-T_{p}}{U \tau}
\end{gathered}
$$

where $r$ is heliocentric distance, $Q_{\{e, p\}}$ represents turbulent heating per unit mass, $\mathbf{q}_{e}$ is the electron heat flux vector (proton heat flux vector has been neglected), and $k_{B}$ is Boltzmann's constant. The $T_{e}-T_{p}$ terms in Eqs. (1)-(2) model Coulomb collisions taking place over a timescale $\tau$.

By using Eqs. (1) and (2), we can study the effects of electrons on the heating of the solar wind. Two possible avenues are available:

1) We may obtain the turbulent heating through the use of a turbulence model [8] and then numerically solve (1) and (2) to compare the results against empirical observations;

2) We may use observations to "invert" (1) and (2) to obtain $Q_{\{e, p\}}$.

We will quickly examine both approaches in this paper. Further details and other results of the two approaches may be found in [11, 12], respectively.

Both approaches rely upon specifying the electron heat flux vector, $\mathbf{q}_{e}$, taken parallel to the magnetic field. (The perpendicular heat flux can be ignored as the magnetic field effectively acts as a thermal blanket, severely 
reducing the heat flux transverse to the field.) A collision dominated heat flux [13] would be inappropriate as collisions do not dominate the solar wind. Collisionless models for the heat flux [14] would be more appropriate, despite the solar wind not being entirely collisionless either. However, the collisionless models can still suffer from missing non-local effects $[15,16]$.

As a result of these difficulties, we adopt an empirical approach. We derive values for $q_{\|}$based on Helios [17] and Ulysses $[18,19]$ observations. The procedure leads to a radial profile for $q_{\|}$for $r<5.4$ AU. Due to the lack of observations farther out, we extrapolate the profile to larger distances but note the obvious caveats in doing so [see 11, 12, for more information on the fits].

\section{TURBULENCE MODELING APPROACH}

Following a von Kármán and Howarth [20] style phenomenology, we write the total turbulent dissipation rate, $Q=Q_{p}+Q_{e}$, as,

$$
Q=\frac{1}{2} \alpha \rho f^{+}\left(\sigma_{c}\right) \frac{Z^{3}}{\lambda}
$$

$\alpha$ is an order-unity constant, $\sigma_{c}$ is the normalized cross helicity, $\lambda$ is the correlation scale (energy containing scale) of the turbulence, and $Z^{2} / 2$ is the total energy available in turbulent fluctuations. $Q$ must be partitioned between the electrons and protons. We accomplish this by defining $Q_{p} \equiv f_{p} Q$ and $Q_{e} \equiv\left(1-f_{p}\right) Q$, with $f_{p}$ clearly being the fraction of heat going into the protons. Observational evidence suggests that near $1 \mathrm{AU}, f_{p} \approx$ $0.60[21,22]$. In principle, $f_{p}$ may be determined by the underlying kinetic physics [23], which operates at scales far smaller than the energy-containing scale described by $\lambda$. This point deserves further study, which is outside the scope of this paper. For now, we simply a adopt a constant $f_{p}=0.60$.

Computing $Q$ requires specifying values for the turbulent quantities $Z^{2}, \lambda$ and $\sigma_{c}$. We obtain these quantities from the following turbulence model [see 8, and references therein];

$$
\begin{gathered}
\frac{\mathrm{d} Z^{2}}{\mathrm{~d} r}=-\frac{Z^{2}}{r}+\frac{C_{\mathrm{sh}}-M \sigma_{D}}{r} Z^{2}+\frac{\dot{E}_{P I}}{U}-\alpha f^{+} \frac{Z^{3}}{\lambda U}, \\
\frac{\mathrm{d} \lambda}{\mathrm{d} r}=\beta f^{+} \frac{Z}{U}-\frac{\beta}{\alpha} \frac{\dot{E}_{P I}}{U Z^{2}} \lambda, \\
\frac{\mathrm{d} \sigma_{c}}{\mathrm{~d} r}=\alpha f^{\prime} \frac{Z}{U \lambda}-\left[\frac{C_{\mathrm{sh}}-M \sigma_{D}}{r}+\frac{\dot{E}_{P I}}{U Z^{2}}\right] \sigma_{c},
\end{gathered}
$$

where $f^{ \pm}\left(\sigma_{c}\right)=\frac{\left(1-\sigma_{c}^{2}\right)^{1 / 2}}{2}\left[\left(1+\sigma_{c}\right)^{1 / 2} \pm\left(1-\sigma_{c}\right)^{1 / 2}\right]$, and $f^{\prime}\left(\sigma_{c}\right)=\sigma_{c} f^{+}-f^{-}$. Driving of the turbulence comes from stream shear, modeled through $C_{\mathrm{sh}}$, and
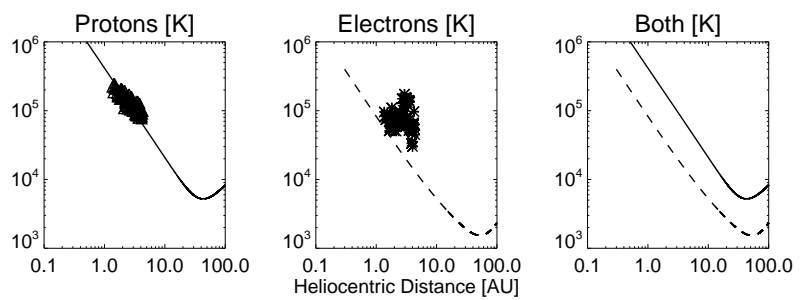

FIGURE 1. Model solutions for the proton and electron temperatures computed without collisions and without electron heat conduction.

pickup protons, $\dot{E}_{P I} . M=1 / 2$ relates to the underlying turbulence geometries and $\sigma_{D}=-1 / 3$ approximates the normalized energy difference (kinetic minus magnetic) of the fluctuations. More details on the model itself, and its parameters, can be found in Breech et al. [8].

We numerically solve the model equations, (4-6), using the results to compute the proton and electron temperatures as per Eqs. (1) and (2). The bounds of the equations run from $0.3 \mathrm{AU}$ to $100 \mathrm{AU}$, though, properly speaking, the heat conduction fits may not apply beyond 5.4 AU. The initial values for the solutions shown below are $Z^{2}=5000(\mathrm{~km} / \mathrm{s})^{2}, \lambda=0.03 \mathrm{AU}$, and $\sigma_{c}=0.6$. Other parameters are set as $U=774 \mathrm{~km} / \mathrm{s}, C_{\mathrm{sh}}=0.25$, $\alpha=2 \beta=0.5$, and $M \sigma_{D}=-1 / 6$. The initial values for the temperatures are taken as $T_{p}=2.0 \times 10^{6} \mathrm{~K}$ and $T_{e}=4.0 \times 10^{5} \mathrm{~K}$, and were chosen because they produce best fits for the observations when all effects are included. Due to space limitations, we only show the temperature solutions [see 8,11 , for more discussions on the turbulence model solutions]. For comparison, we use temperature values observed by Ulysses in the high latitude, fast solar wind. We use the total electron temperature, rather than the core, halo or strahl populations, as the turbulence should heat all electrons.

Figure 1 displays the temperature solutions without heat conduction and almost without collisions. The collision timescale $\tau$ was set equal to the time for plasma to transit to $100 \mathrm{AU}$, which effectively removes collision effects from the results. The proton solution agrees very well with the observed data, but the electron solution misses almost all the data.

Turning on heat conduction and allowing collisions ( $\tau=$ transit time to $10 \mathrm{AU}$ ), produces interesting results as shown in Fig. 2. Both effects are important, but in different regions. Compared to Figure 1 collisions attempt to equalize the temperatures beyond $10 \mathrm{AU}$, while heat conduction produces higher temperatures near 3-5 AU. [see 11, for more information on this point]. The electron temperature now features a "shelf" region between 1 and $10 \mathrm{AU}$ where $T_{e}(r)$ does not decrease as rapidly as it does outside that region. Interestingly, the electron temperature solution crosses the proton temperature so- 

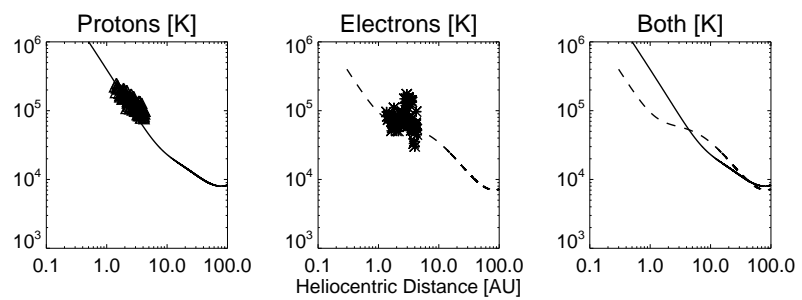

FIGURE 2. Model solutions for the proton and electron temperatures computed with both collisions and electron heat conduction turned on. The proton solution remains reasonable. The electron solution displays a "shelf-like" region between 1 and $10 \mathrm{AU}$.

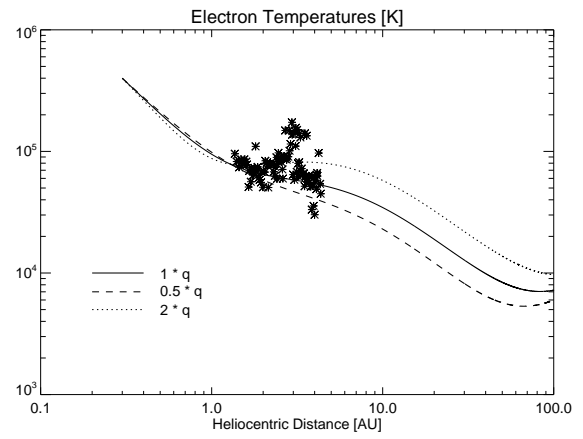

FIGURE 3. Solutions for the electron temperature using different profiles of the heat flux vector, $q_{\|}$.

lution near $5 \mathrm{AU}$, after which the electrons are actually warmer than the protons. Collisions then begin to take hold, resulting in higher proton temperatures and lower electron temperatures.

The shelf is somewhat sensitive to the underlying electron heat flux vector, $q_{\|}$, used to compute the solutions. Figure 3 shows the electron temperature solutions computed using three different profiles for $q_{\|}$. The baseline profile is the observationally derived profile used in the solutions shown in Figure 2. The other two solutions use a heat flux vector where the baseline $q_{\|}$was multiplied by 2 and divided by 2 . Since Scime et al. [19] found small variations in the heat flux with latitude, any variation in the heat flux measurements should lie between these two extremes. The electron temperature solutions show that increasing the heat flux makes the shelf more pronounced. Lowering the heat flux lessens the shelf.

\section{IN-SITU DATA ANALYSIS APPROACH}

Rather than use a turbulence model, we can use observations to "invert" Eqs. (1) and (2) to obtain $Q_{\{e, p\}}$. Figure 4 shows observations of $T_{p}$ and $T_{e}$ taken from the fast wind solar wind data $(U>600 \mathrm{~km} / \mathrm{s})$ of Helios and Ulysses. Best fit curves are also shown. To avoid the larger number of Ulysses data points overwhelming the

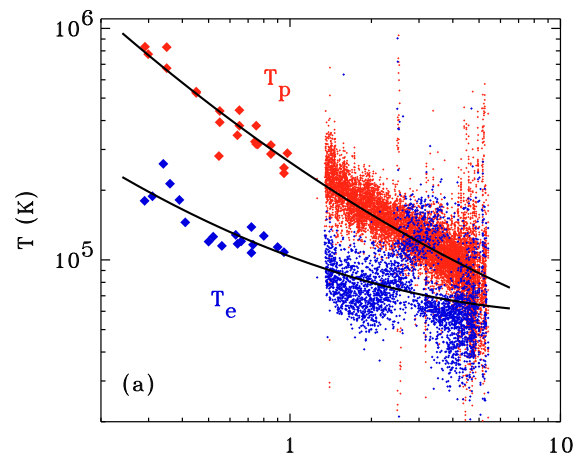

FIGURE 4. Observed electron and proton temperatures from Helios and Ulysses along with best fit curves.

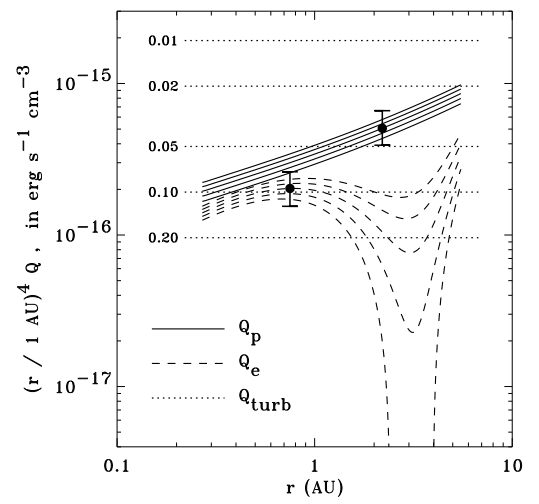

FIGURE 5. Empirically derived heating rates for protons (solid lines) and electrons (dashed lines) in the fast solar wind, with multiple curves showing results for $u=600,650,700$, 750 , and $800 \mathrm{~km} \mathrm{~s}^{-1}$ (from bottom to top for each set of curves). Shown for comparison is $Q_{\text {turb }}$, an approximation to the turbulent heating rate [12], for different values of $\lambda$.

Helios data points in the least-squares fitting process, the data sets from the two spacecraft were weighted equally.

We solved Eqs. (1) and (2) for the volumetric heating rates $Q_{p}$ and $Q_{e}$ over the range of heliocentric distances covered by the Helios and Ulysses measurements. An interesting aspect of this approach is that because the internal energy equations are not being solved for the temperatures-but instead for the heating rates-we can avoid complicated numerical differential equation techniques and use a straightforward algebraic solution for $Q_{p}$ and $Q_{e}$. Even the radial derivatives can be computed analytically from the fits given in Figure 4.

Figure 5 shows the results, plotted as $r^{4} Q_{p}(r)$ and $r^{4} Q_{e}(r)$ rather than the rates themselves, because the latter decrease very steeply with distance. Removing the dominant radial variation makes the subtle relative differences between $Q_{p}$ and $Q_{e}$ easier to see. Clearly $Q_{p}$ can be approximated by a power law, with $Q_{p} \propto r^{-3.5}$. Although not shown, the turbulence model discussed 


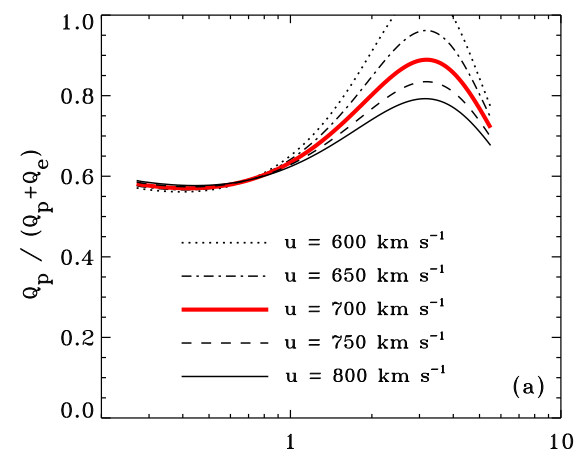

FIGURE 6. Ratios of the proton heating rate to the total (proton + electron) heating rate.

above produces results consistent with such a scaling. Note that it is not possible to fit the electron heating rate $Q_{e}(r)$ with a simple power-law function because of the nonlocal heat conduction.

Figure 6 shows the ratio of $Q_{p} /\left(Q_{p}+Q_{e}\right)$, as it varies with wind speed. This is the $f_{p}$ ratio used in partitioning the turbulent heating computed for the turbulence model. The ratio at $r \leq 1 \mathrm{AU}$ appears to be insensitive to the wind speed because both $Q_{p}$ and $Q_{e}$ vary linearly with the bulk flow speed at these distances. Near $1 \mathrm{AU}, f_{p} \approx$ 0.60 , consistent with other observations [21, 22]. Further out, $f_{p}$ shows an increased spread, which is the result of the electron heat conduction having a larger relative impact on $Q_{e}$ as the wind speed changes. $f_{p}$ can rise above $80 \%$ at these distances before apparently relaxing back towards $60 \%$. It is unknown what behavior $f_{p}$ may have beyond 5.4 AU.

\section{CONCLUSIONS}

In this paper, we have explored the effects of electrons and protons on the heating of the solar wind. We summarized two different, but complementary, approaches; 1) modeling of the solar wind turbulence and then comparing against the observed electron and proton temperatures; and 2) in-situ data analysis by using the observations to derive the heating rates for the electrons and protons. More details of these approaches can be found in Breech et al. [11] and Cranmer et al. [12], respectively.

In general, we find that observations are reasonably explained with $\sim 60 \%$ of the turbulent dissipation to heat the protons at $1 \mathrm{AU}$, weak collisional coupling between the protons and electrons (at least to around $10 \mathrm{AU}$ ), and a reasonable fit for the observed electron heat flux vector. The amount of heating that goes into the protons increases significantly between 2 and 5 AU. Modelling results suggest the existence of a "shelf" region in the electron temperature at those same distances. The exact details of these results are subject to uncertainties in the heat conduction fits.

\section{ACKNOWLEDGMENTS}

BAB's research was supported by an appointment to the NASA Postdoctoral Program at Goddard Space Flight Center. SRC's work was supported by the NASA under grants NNG04GE77G, NNX06AG95G, and NNX09AB27G to the Smithsonian Astrophysical Observatory. WHM's research was supported by NSF ATM 0752135 (SHINE) and NASA NNX08AI47G (Heliophysics Theory Program). JCK's research was supported in part by NASA grant NNX08AW07G.

\section{REFERENCES}

1. P. J. Coleman, Astrophys. J. 153, 371-388 (1968).

2. B. T. MacBride, C. W. Smith, and M. A. Forman, Astrophys. J. 679, 1644-1660 (2008).

3. R. Marino, L. Sorriso-Valvo, V. Carbone, A. Noullez, R. Bruno, and B. Bavassano, Astrophys. J. 677, L71-L74 (2008).

4. S. R. Cranmer, A. A. van Ballegooijen, and R. Edgar, Astrophys. J. Suppl. Ser 171, 520-551 (2007).

5. E. Marsch, and C.-Y. Tu, J. Geophys. Res. 98, 21045 (1993).

6. S. Oughton, and W. H. Matthaeus, J. Geophys. Res. 100, 14 783-14 799 (1995).

7. G. P. Zank, W. H. Matthaeus, and C. W. Smith, $J$. Geophys. Res. 101, 17 093-17 107 (1996).

8. B. Breech, W. H. Matthaeus, J. Minnie, J. W. Bieber, S. Oughton, C. W. Smith, and P. A. Isenberg, J. Geophys. Res. 113 (2008).

9. J. L. Kohl, et al., Astrophys. J. 501, L127 (1998).

10. J. C. Kasper, A. J. Lazarus, and S. P. Gary, Phys. Rev. Lett. 101, 261103 (2008).

11. B. Breech, W. H. Matthaeus, S. R. Cranmer, J. C. Kasper, and S. Oughton, J. Geophys. Res. 114 (2009).

12. S. Cranmer, W. Matthaeus, B. Breech, and J. Kasper, Astrophys. J. 702, 1604-1614 (2009).

13. L. Spitzer, and R. Härm, Phys. Rev. 89, 977-981 (1953).

14. J. V. Hollweg, J. Geophys. Res. 81, 1649-1658 (1976).

15. M. V. Canullo, A. Costa, and C. Ferro-Fontan, Astrophys. J. 462, 1005-1010 (1996).

16. J. D. Scudder, and S. Olbert, J. Geophys. Res. 84, 2755-2772 (1979).

17. W. Pilipp, H. Miggenrieder, K. Mühlhäuser, H. Rosenbauer, and R. Schwenn, J. Geophys. Res. 95, 6305-6329 (1990).

18. E. E. Scime, S. J. Bame, W. C. Feldman, S. P. Gary, J. L. Phillips, and A. Balogh, J. Geophys. Res. 99. 23401-23410 (1994).

19. E. E. Scime, A. E. Badeau Jr, and J. Littleton, Geophys. Res. Lett. 26, 2129-2132 (1999).

20. T. von Kármán, and L. Howarth, Proc. Roy. Soc. London Ser. A 164, 192-215 (1938).

21. R. J. Leamon, W. H. Matthaeus, C. W. Smith, and H. K. Wong, Astrophys. J. 507, L181-L184 (1998).

22. R. J. Leamon, C. W. Smith, N. F. Ness, and H. K. Wong, J. Geophys. Res. 104, 22331 (1999).

23. S. P. Gary, and J. E. Borovsky, J. Geophys. Res. 109, 6105 (2004). 